\title{
ALMOST-ARITHMETIC PROGRESSIONS AND UNIFORM DISTRIBUTION
}

\author{
BY \\ H. NIEDERREITER
}

\begin{abstract}
In a recent paper, P. E. O'Neil gave a new criterion for uniform distribution modulo one in terms of almost-arithmetic progressions. We investigate the relation between almost-arithmetic progressions and uniformly distributed sequences from a quantitative point of view. An upper bound for the discrepancy of almost-arithmetic progressions is given which is shown to be best possible. Estimates for more general sequences are also obtained. As an application, we prove a quantitative form of Fejér's theorem on the uniform distributivity of slowly increasing sequences.
\end{abstract}

1. Introduction. This paper has its origin in a quantitative analysis of a result given by P. E. O'Neil [3] which revealed the strong relation between almostarithmetic progressions and uniformly distributed sequences. The natural approach to a quantitative study is by means of the concept of discrepancy. As a first step, we give upper bounds for the discrepancy of almost-arithmetic progressions (\$2). A method is employed which interprets the estimation of discrepancy as a convex program, and which is described in detail in [2]. Subsequently, we turn to more general sequences and obtain estimates for their discrepancy based on the decomposition of such sequences into almost-arithmetic progressions (\$3). Thus, the cumbersome task of computing the discrepancy of a sequence can, at least theoretically, be reduced to the following two problems: (i) find a decomposition of the sequence into almost-arithmetic progressions; (ii) find sharp estimates for the discrepancy of almost-arithmetic progressions. Problem (ii) is answered here with optimal accuracy, possibilities for solving (i) were exhibited by P. E. O'Neil [3] in the context of specific examples. In the last section, the general results of this paper are applied successfully to estimate the discrepancy of a class of sequences which includes a number of classical examples of uniformly distributed sequences.

The two definitions and the theorem given below will be fundamental for our discussion. For an account of the theory of uniform distribution modulo one, the reader is referred to [1].

Definition 1.1 (O’NeIL [3]). For $\delta \geqq 0$ and $\varepsilon>0$, a sequence $a_{1}<a_{2}<\cdots<a_{N}$ of points from the interval $[0,1]$ is called an almost-arithmetic progression- $(\delta, \varepsilon)$

Received by the editors November 16, 1970.

AMS 1970 subject classifications. Primary 10F40; Secondary 90C25.

Key words and phrases. Almost-arithmetic progressions, uniform distribution modulo one, discrepancy, convex programming.

Copyright (C) 1971, American Mathematical Society 
if there exists an $\eta, 0<\eta \leqq \varepsilon$, such that

(1) $0 \leqq a_{1} \leqq \eta+\delta \eta$,

(2) $\eta-\delta \eta \leqq a_{k+1}-a_{k} \leqq \eta+\delta \eta$ for $1 \leqq k \leqq N-1$,

(3) $1-\eta-\delta \eta \leqq a_{N} \leqq 1$.

TheOREM 1.1 (O'NeIL [3]). The sequence $a_{1}, a_{2}, \ldots, a_{N}, \ldots$ of points in $[0,1]$ is uniformly distributed modulo one iff the following condition holds: given $\delta, \varepsilon$, and $\varepsilon^{\prime}$ all positive, there exists an $\bar{N}$ such that, for $N>\bar{N}$, the partial sequence $a_{1}, a_{2}, \ldots, a_{N}$ can be partitioned into a set of disjoint almost-arithmetic progressions- $(\delta, \varepsilon)$ with at most $N_{0}$ points left over, $N_{0}<\varepsilon^{\prime} N$.

Definition 1.2. Given a sequence $a_{1}, a_{2}, \ldots, a_{N}$ of points in $[0,1]$, for $M \subset[0,1]$, let $A(M ; N)$ be the number of $a_{i}, 1 \leqq i \leqq N$, contained in $M$. Then the discrepancy $D_{N}$ of the given sequence is defined by

$$
D_{N}=\sup _{0<\alpha \leqq 1}\left|\frac{A([0, \alpha) ; N)}{N}-\alpha\right| .
$$

2. Discrepancy of almost-arithmetic progressions. We consider a finite sequence in $[0,1]$ whose elements are ordered according to their magnitude: $0 \leqq a_{1} \leqq a_{2} \leqq \cdots$ $\leqq a_{N} \leqq 1$. It was shown in [2] that, in this case, we can write $D_{N}$ in a simpler form:

$$
D_{N}=\max _{i=1, \ldots, N} \max \left(\left|\frac{i}{N}-a_{i}\right|,\left|\frac{i-1}{N}-a_{i}\right|\right) \text {. }
$$

In the presence of linear inequalities for the $a_{i}$, the problem of estimating the discrepancy can be viewed as a convex program. Thus, it suffices to evaluate a certain target function at the vertices of the convex polytope defined by the given system of linear inequalities (see [2] for a thorough description of this method).

To be more specific, let us consider an almost-arithmetic progression- $(\delta, \varepsilon)$, say $a_{1}, a_{2}, \ldots, a_{N}$. We assume $\delta<1$; then the condition $a_{1}<a_{2}<\cdots<a_{N}$ follows automatically from (2). The convex polytope in question is the following: the set $P$ of all $\left(x_{1}, \ldots, x_{N}\right) \in R^{N}$ satisfying

(5 $0 \leqq x_{1} \leqq \eta+\delta \eta$

$\left(5_{2}\right)-\left(5_{N}\right) \eta-\delta \eta \leqq x_{j+1}-x_{j} \leqq \eta+\delta \eta, 1 \leqq j \leqq N-1$, $\left(5_{N+1}\right) 1-\eta-\delta \eta \leqq x_{N} \leqq 1$.

The target function $f\left(x_{1}, \ldots, x_{N}\right)$ to be maximized is given by

$$
f\left(x_{1}, \ldots, x_{N}\right)=\max _{i=1, \ldots, N} \max \left(\left|\frac{i}{N}-x_{i}\right|,\left|\frac{i-1}{N}-x_{i}\right|\right) .
$$

THEOREM 2.1. Let $a_{1}, a_{2}, \ldots, a_{N}$ be an almost-arithmetic progression- $(\delta, \varepsilon)$ with $0 \leqq \delta<1$ and $\varepsilon>0$, let $\eta$ be the positive real number corresponding to the sequence according to Definition 1.1, and let $D_{N}$ be the discrepancy of the sequence. Then

$$
\begin{array}{ll}
D_{N} \leqq 1 / N+\delta /\left(1+\sqrt{ }\left(1-\delta^{2}\right)\right) & \text { for } \delta>0, \\
D_{N} \leqq \min (\eta, 1 / N) & \text { for } \delta=0 .
\end{array}
$$


Proof. We write $f\left(x_{1}, \ldots, x_{N}\right)=\max _{i=1, \ldots, N} g_{i}\left(x_{1}, \ldots, x_{N}\right)$ with

$$
g_{i}\left(x_{1}, \ldots, x_{N}\right)=\max \left(\left|i / N-x_{i}\right|,\left|(i-1) / N-x_{i}\right|\right) .
$$

The maximum of $g_{i}$ is attained at an extreme value of $x_{i}$. Therefore, we shall first maximize and minimize the coordinate $x_{i}$ over the convex polytope $P$, the extreme values being attained at vertices of $P$. Note that the boundary of $P$ is determined by $N+1$ classes (in fact, pairs) of parallel hyperplanes:

(7 $\left.x_{1}\right)=0, x_{1}=\eta+\delta \eta$,

$\left(7_{2}\right)-\left(7_{N}\right) x_{j+1}-x_{j}=\eta-\delta \eta, x_{j+1}-x_{j}=\eta+\delta \eta, 1 \leqq j \leqq N-1$,

$\left(7_{N+1}\right) x_{N}=1-\eta-\delta \eta, x_{N}=1$.

We get all vertices by the following procedure: choose one hyperplane from each of $N$ classes $\left(7_{1}\right), \ldots,\left(7_{k-1}\right),\left(7_{k+1}\right), \ldots,\left(7_{N+1}\right)$, find the unique point of intersection, and check whether this point satisfies the remaining defining condition $\left(5_{k}\right)$ of $P$. To have a shorter denotation, let $\alpha$ stand for one of 0 and $\eta+\delta \eta$, and let $\beta$ stand for one of $1-\eta-\delta \eta$ and 1 . We exclude the case $\delta=0$ for the time being. We distinguish four cases in terms of $k$. The subscript $i, 1 \leqq i \leqq N$, is assumed to be fixed, therefore we take the liberty of suppressing the dependence on $i$ in some of the constants.

Case 1. $k=1$. Using $\left(7_{2}\right)-\left(7_{N+1}\right)$, each choice of $\left(\varepsilon_{1}, \ldots, \varepsilon_{N-1}\right)$ with $\varepsilon_{j}=0$ or 1 , $1 \leqq j \leqq N-1$, leads to a point $\left(x_{1}, \ldots, x_{N}\right)$ with

$$
x_{i}=\beta-(N-i) \eta+\delta \eta\left((-1)^{\varepsilon_{i}}+\cdots+(-1)^{\varepsilon_{N-1}}\right) .
$$

Condition $\left(5_{1}\right)$ requires

$$
0 \leqq \beta-(N-1) \eta+\delta \eta\left((-1)^{\varepsilon_{1}}+\cdots+(-1)^{\varepsilon_{N-1}}\right) \leqq \eta+\delta \eta
$$

or

$$
A=\frac{(N-1) \eta-\beta}{\delta \eta} \leqq(-1)^{\varepsilon_{1}}+\cdots+(-1)^{\varepsilon_{N-1}} \leqq \frac{N \eta+\delta \eta-\beta}{\delta \eta}=B
$$

$x_{i}$ is maximal or minimal according as a maximal or minimal number of $\varepsilon_{j}, 1 \leqq j$ $\leqq N-1$, are zero. Thus, let $a$ be the number of $\varepsilon_{j}, 1 \leqq j \leqq N-1$, with $\varepsilon_{j}=0$, and let $b$ be the number of $\varepsilon_{j}, 1 \leqq j \leqq N-1$, with $\varepsilon_{j}=1$. Then $a-b=(-1)^{\varepsilon_{1}}+\cdots+(-1)^{\varepsilon_{N-1}}$ $\leqq B$; on the other hand, $a+b=N-1$, hence $a \leqq(B+N-1) / 2$. Furthermore, $(B+N-1) / 2=(N(\eta+\delta \eta)-\beta) / 2 \delta \eta \geqq 0$, since $\beta=x_{N} \leqq N(\eta+\delta \eta)$. Putting $a^{\prime}$ $=\min (N-i,[(B+N-1) / 2])$, we have then

$\left(10^{\prime}\right) \max x_{i}=\beta-(N-i) \eta+\delta \eta\left(a^{\prime}-\left(N-i-a^{\prime}\right)\right)=\beta+2 a^{\prime} \delta \eta-(N-i)(\eta+\delta \eta)$.

In the expressions max $x_{i}$ and min $x_{i}$, the maximum or minimum is meant to be over all vertices of $P$ belonging to the case under consideration, unless explicitly stated otherwise.

Turning to the minimum, we have $a-b \geqq A$, or $b \leqq(N-1-A) / 2$. Again, 
$(N-1-A) / 2=(\beta-(N-1)(\eta-\delta \eta)) / 2 \delta \eta \geqq 0$, since $\beta=x_{N} \geqq(N-1)(\eta-\delta \eta)$. Putting $b^{\prime}=\min (N-i,[(N-1-A) / 2])$, we have

$\left(11^{\prime}\right) \min x_{i}=\beta-(N-i) \eta+\delta \eta\left(-b^{\prime}+\left(N-i-b^{\prime}\right)\right)=\beta-2 b^{\prime} \delta \eta-(N-i)(\eta-\delta \eta)$.

The remaining three cases are treated in a completely similar manner. Therefore, we just list the statements corresponding to $\left(8^{\prime}\right),\left(9^{\prime}\right),\left(10^{\prime}\right)$, and $\left(11^{\prime}\right)$ for each of those cases.

Case 2. $1<k \leqq i$.

$$
\begin{gathered}
x_{i}=\beta-(N-i) \eta+\delta \eta\left((-1)^{\varepsilon_{i}}+\cdots+(-1)^{\varepsilon_{N}-1}\right), \\
C=(\alpha-\beta+(N-1) \eta) / \delta \eta-1 \\
\leqq \\
\leqq(-1)^{\varepsilon_{1}}+\cdots+(-1)^{\varepsilon_{k}-2}+(-1)^{\varepsilon_{k}}+\cdots+(-1)^{\varepsilon_{N-1}} \\
\max x_{i}=\beta+2 a^{\prime \prime} \delta \eta-(N-i)(\eta+\delta \eta) \\
\quad \text { with } a^{\prime \prime}=\min (N-i,[(D+N-2) / 2]), \\
\min x_{i}=\beta-2 b^{\prime \prime} \delta \eta-(N-i)(\eta-\delta \eta) \\
\text { with } b^{\prime \prime}=\min (N-i,[(N-2-C) / 2]) .
\end{gathered}
$$

Case 3. $i<k \leqq N$.

$$
\begin{gathered}
x_{i}=\alpha+(i-1) \eta+\delta \eta\left((-1)^{\varepsilon_{1}}+\cdots+(-1)^{\varepsilon_{i}-1}\right), \\
E=(\beta-\alpha-(N-1) \eta) / \delta \eta-1 \\
\leqq \\
\leqq \\
(\beta-\alpha)^{\varepsilon_{1}}+\cdots+(-1)^{\varepsilon_{k-2}}+(-1)^{\varepsilon_{k}}+\cdots+(-1)^{\varepsilon_{N-1}} \\
\max x_{i}=\alpha+2 a^{m} \delta \eta+(i-1)(\eta-\delta \eta) / \delta \eta+1=F, \\
\text { with } a^{m}=\min (i-1,[(F+N-2) / 2]), \\
\min x_{i}=\alpha-2 b^{m} \delta \eta+(i-1)(\eta+\delta \eta) \\
\text { with } b^{m}=\min (i-1,[(N-2-E) / 2]) .
\end{gathered}
$$

Case 4. $k=N+1$.

$$
\begin{gathered}
x_{i}=\alpha+(i-1) \eta+\delta \eta\left((-1)^{\varepsilon_{1}}+\cdots+(-1)^{\varepsilon_{i}-1}\right), \\
G=(1-\alpha-N \eta-\delta \eta) / \delta \eta \leqq(-1)^{\varepsilon_{1}}+\cdots+(-1)^{\varepsilon_{N-1}} \\
\leqq(1-\alpha-(N-1) \eta) / \delta \eta=H, \\
\max x_{i}=\alpha+2 a^{(4)} \delta \eta+(i-1)(\eta-\delta \eta) \\
\text { with } a^{(4)}=\min (i-1,[(H+N-1) / 2]), \\
\min x_{i}=\alpha-2 b^{(4)} \delta \eta+(i-1)(\eta+\delta \eta) \\
\text { with } b^{(4)}=\min (i-1,[(N-1-G) / 2]) .
\end{gathered}
$$

Because of $D-1 \leqq B$ we have $a^{\prime \prime} \leqq a^{\prime}$; similarly, $C+1 \leqq A$ implies $b^{\prime \prime} \leqq b^{\prime}$. Therefore, Case 2 can be dropped. For similar reasons, Case 3 can be dropped in favor of Case 4. 
Let us look at $\left(10^{\prime}\right)$ and $\left(10^{(4)}\right)$. Putting $\bar{a}^{\prime}=\min (N-i,(B+N-1) / 2)$ and $\bar{a}^{(4)}=\min (i-1,(H+N-1) / 2)$, we have

$$
\begin{aligned}
& \max x_{i} \leqq \beta+2 \bar{a}^{\prime} \delta \eta-(N-i)(\eta+\delta \eta), \\
& \max x_{i} \leqq \alpha+2 \bar{a}^{(4)} \delta \eta+(i-1)(\eta-\delta \eta) .
\end{aligned}
$$

It is easily seen that the value of the expression on the right-hand side of $\left(12^{\prime}\right)$ corresponding to $\beta=1-\eta-\delta \eta$ is less than or equal to the value of the same expression corresponding to $\beta=1$. Thus

$$
\max x_{i} \leqq 1+2 \delta \eta \min (N-i,(N-1) / 2+(N \eta+\delta \eta-1) / 2 \delta \eta)-(N-i)(\eta+\delta \eta) .
$$

For $\left(12^{(4)}\right), \alpha=\eta+\delta \eta$ yields the value which is possibly larger:

$$
\begin{aligned}
\max x_{i} \leqq & \eta+\delta \eta+2 \delta \eta \min (i-1,(N-1) / 2+(1-N \eta-\delta \eta) / 2 \delta \eta) \\
& +(i-1)(\eta-\delta \eta) .
\end{aligned}
$$

Put $T=(N-1) / 2+(1-N \eta-\delta \eta) / 2 \delta \eta$. Using $\min (c-d, c-e)=c-\max (d, e)$ in $\left(13^{\prime}\right)$, we can write $\left(13^{\prime}\right),\left(13^{(4)}\right)$ in the form:

$\left(14^{(4)}\right) \quad \max x_{i} \leqq \eta+\delta \eta+(i-1)(\eta-\delta \eta)+2 \delta \eta \min (i-1, T)$.

If $i-1 \leqq T$, then the right-hand sides of $\left(14^{\prime}\right),\left(14^{(4)}\right)$ are both $i(\eta+\delta \eta)$; therefore,

$$
\max x_{i} \leqq i(\eta+\delta \eta) \text { for } i-1 \leqq T .
$$

If $i-1>T$, then the right-hand sides of $\left(14^{\prime}\right),\left(14^{(4)}\right)$ are both $1-(N-i)(\eta-\delta \eta)$; therefore,

$$
\max x_{i} \leqq 1-(N-i)(\eta-\delta \eta) \text { for } i-1>T .
$$

In both (15) and (16), the maximum is extended over all vertices of $P$. Let us now look at $\left|x_{i}-i / N\right|$. In accordance with the above results, we shall distinguish two cases:

Case I. $i \leqq T+1=(1-N(\eta-\delta \eta)) / 2 \delta \eta$. This case can only occur if $T+1 \geqq 0$, i.e. if $N \eta \leqq 1 /(1-\delta)$. We have then $x_{i}-i / N \leqq i(\eta+\delta \eta-1 / N)$. We can assume $\eta+\delta \eta$ $-1 / N \geqq 0$, for otherwise all $x_{i}-i / N$ are negative, hence $\max \left|x_{i}-i / N\right|$ will occur at the minimal value of $x_{i}$. Thus $N \eta \geqq 1 /(1+\delta)$, hence $1 /(1+\delta) \leqq N \eta \leqq 1 /(1-\delta)$. Furthermore,

$$
x_{i}-\frac{i}{N} \leqq i\left(\eta+\delta \eta-\frac{1}{N}\right) \leqq \frac{1-N \eta(1-\delta)}{2 \delta \eta}\left(\eta+\delta \eta-\frac{1}{N}\right)=\frac{2 N \eta-1-N^{2} \eta^{2}\left(1-\delta^{2}\right)}{2 N \delta \eta} .
$$

Set $N \eta=t$, and $h(t)=\left(2 t-1-t^{2}\left(1-\delta^{2}\right)\right) / 2 \delta t$. Since $h(t)$ has an absolute maximum in the interval $[1 /(1+\delta), 1 /(1-\delta)]$ at $t_{0}=1 / \sqrt{ }\left(1-\delta^{2}\right)$, we get

$$
x_{i}-i / N \leqq h\left(t_{0}\right)=\delta /\left(1+\sqrt{ }\left(1-\delta^{2}\right)\right) .
$$


Case II. $i>T+1=(1-N(\eta-\delta \eta)) / 2 \delta \eta$. This case can only occur if $T+1 \leqq N$, i.e. if $N \eta \geqq 1 /(1+\delta)$. We have $x_{i}-i / N \leqq 1-(N-i)(\eta-\delta \eta)-i / N=(N-i)(1 / N+\delta \eta-\eta)$. For the same reason as above, we can assume $1 / N+\delta \eta-\eta \geqq 0$, or $N \eta \leqq 1 /(1-\delta)$. Therefore $1 /(1+\delta) \leqq N \eta \leqq 1 /(1-\delta)$. Furthermore,

$$
\begin{aligned}
x_{i}-\frac{i}{N} & \leqq(N-i)\left(\frac{1}{N}+\delta \eta-\eta\right) \leqq\left(N-\frac{1-N \eta(1-\delta)}{2 \delta \eta}\right)\left(\frac{1}{N}+\delta \eta-\eta\right) \\
& =\frac{2 N \eta-1-N^{2} \eta^{2}\left(1-\delta^{2}\right)}{2 N \delta \eta} \leqq \frac{\delta}{1+\sqrt{ }\left(1-\delta^{2}\right)} .
\end{aligned}
$$

As to the minimum, we have to start from $\left(11^{\prime}\right)$ and $\left(11^{(4)}\right)$. We get estimates from below for $\min x_{i}$ by replacing $b^{\prime}, b^{(4)}$ by $\bar{b}^{\prime}=\min (N-i,(N-1-A) / 2)$ and $\bar{b}^{(4)}$ $=\min (i-1,(N-1-G) / 2)$, respectively. Working in the same way as above through the statements corresponding to $\left(13^{\prime}\right),\left(13^{(4)}\right),\left(14^{\prime}\right)$, and $\left(14^{(4)}\right)$, we finally arrive at

$$
\begin{gathered}
\min x_{i} \geqq(i-1)(\eta-\delta \eta) \text { for } i-1 \leqq U, \\
\min x_{i} \geqq 1-(N-i+1)(\eta+\delta \eta) \text { for } i-1>U,
\end{gathered}
$$

with $U=(N-1) / 2-(1-N \eta-\delta \eta) / 2 \delta \eta$. The minimum in (17), (18) is meant to be over all vertices of $P$. In the same fashion as in Case I and Case II from above we obtain $(i-1) / N-x_{i} \leqq \delta /\left(1+\sqrt{ }\left(1-\delta^{2}\right)\right)$. Therefore

$$
-\frac{1}{N}-\frac{\delta}{1+\sqrt{ }\left(1-\delta^{2}\right)} \leqq x_{i}-\frac{i}{N} \leqq \frac{\delta}{1+\sqrt{ }\left(1-\delta^{2}\right)}
$$

and

$$
-\frac{\delta}{1+\sqrt{ }\left(1-\delta^{2}\right)} \leqq x_{i}-\frac{i-1}{N} \leqq \frac{1}{N}+\frac{\delta}{1+\sqrt{ }\left(1-\delta^{2}\right)}
$$

hence

$$
\max \left(\left|\frac{i}{N}-x_{i}\right|,\left|\frac{i-1}{N}-x_{i}\right|\right) \leqq \frac{1}{N}+\frac{\delta}{1+\sqrt{ }\left(1-\delta^{2}\right)} \quad \text { for all } i, 1 \leqq i \leqq N,
$$

which yields the desired estimate for $D_{N}$.

It remains to consider the case $\delta=0$. We have $x_{i}=x_{1}+(i-1) \eta$, hence $x_{i}-(i-1) / N$ $=x_{1}+(i-1)(\eta-1 / N)$. If $\eta \geqq 1 / N$, then $0 \leqq x_{i}-(i-1) / N \leqq x_{1}+(N-1)(\eta-1 / N)$ $=x_{N}-1+1 / N \leqq 1 / N$ and $-1 / N \leqq x_{i}-i / N \leqq 0$. If $\eta<1 / N$, then $\eta \geqq x_{i}-(i-1) / N$ $\geqq x_{1}+(N-1)(\eta-1 / N)=x_{N}-1+1 / N \geqq-\eta+1 / N$ and $\eta-1 / N \geqq x_{i}-i / N \geqq-\eta$. In any case, we have

$$
\max \left(\left|i / N-x_{i}\right|,\left|(i-1) / N-x_{i}\right|\right) \leqq \min (\eta, 1 / N) \text { for all } i, 1 \leqq i \leqq N,
$$

and so $D_{N} \leqq \min (\eta, 1 / N)$. The proof is complete. 
The given estimate is, in fact, best possible. This is illustrated by the following example. The sequence:

$$
\frac{2}{15}, \frac{4}{15}, \frac{2}{5}, \frac{8}{15}, \frac{2}{3}, \frac{7}{10}, \frac{11}{15}, \frac{23}{30}, \frac{4}{5}, \frac{5}{6}, \frac{13}{15}, \frac{9}{10}, \frac{14}{15}, \frac{29}{30}, 1
$$

is an almost-arithmetic progression- $(\delta, \varepsilon)$ with $\eta=1 / 12, \delta=3 / 5$, and $N=15$. We have $1 / N+\delta /\left(1+\sqrt{ }\left(1-\delta^{2}\right)\right)=2 / 5$. On the other hand, $|(1 / N) A([0,2 / 3) ; N)-2 / 3|$ is also $2 / 5$.

We may also give an estimate depending solely on the parameters $\delta$ and $\eta$, by just estimating the number $N$ of elements in the sequence in terms of $\delta$ and $\eta$ :

COROLlaRY. If $\eta+\delta \eta<1$, then

$$
D_{N} \leqq(\eta+\delta \eta) /(1-\eta-\delta \eta)+\delta /\left(1+\sqrt{ }\left(1-\delta^{2}\right)\right) .
$$

Proof. This follows from $1-\eta-\delta \eta \leqq x_{N} \leqq N(\eta+\delta \eta)$.

3. More general sequences. Having established a sharp upper bound for the discrepancy of almost-arithmetic progressions, we now proceed to estimate the discrepancy of sequences which essentially are obtained by superposition of almostarithmetic progressions. To this end, we first prove the following

LEMMA 3.1. For $1 \leqq i \leqq k$, let $\omega_{i}$ be a sequence of $N_{i}$ elements from the interval $[0,1]$ with discrepancy $D_{N_{i}}^{(i)}$. Let $\omega$ be a superposition of $\omega_{1}, \ldots, \omega_{k}$, i.e. a sequence consisting of the elements of the $\omega_{i}$ in some order, and set $N=N_{1}+\cdots+N_{k}$. Then, for the discrepancy $D_{N}$ of $\omega$ we have $D_{N} \leqq \sum_{i=1}^{k}\left(N_{i} / N\right) D_{N_{i}}^{(i)}$.

Proof. For $0<\alpha \leqq 1$, let $A\left(\alpha ; N_{i} ; \omega_{i}\right)$ (resp. $A(\alpha ; N ; \omega)$ ) denote the counting function $A\left([0, \alpha) ; N_{i}\right)$ (resp. $A([0, \alpha) ; N)$ ) with respect to the sequence $\omega_{i}$ (resp. $\left.\omega\right)$. Then $A(\alpha ; N ; \omega)=\sum_{i=1}^{k} A\left(\alpha ; N_{i} ; \omega_{i}\right)$. Hence

$$
\left|\frac{A(\alpha ; N ; \omega)}{N}-\alpha\right|=\left|\sum_{i=1}^{k} \frac{N_{i}}{N}\left(\frac{A\left(\alpha ; N_{i} ; \omega_{i}\right)}{N_{i}}-\alpha\right)\right| \leqq \sum_{i=1}^{k} \frac{N_{i}}{N} D_{N_{i}}^{(i)} .
$$

THEOREM 3.1. Let $\omega$ be a sequence of $N$ elements from the interval $[0,1]$ which satisfies the following condition: $\omega$ can be partitioned into $k$ almost-arithmetic progressions- $\left(\delta_{i}, \varepsilon_{i}\right), \delta_{i} \leqq \delta<1$, having $N_{i}$ elements, with $N_{0}$ elements left over. Then the discrepancy $D_{N}$ of $\omega$ satisfies

$$
D_{N} \leqq \frac{k}{N}+\sum_{i=1}^{k} \frac{N_{i}}{N} \frac{\delta_{i}}{1+\sqrt{ }\left(1-\delta_{i}^{2}\right)}+\frac{N_{0}}{N} \leqq \frac{k}{N}+\frac{N-N_{0}}{N} \frac{\delta}{1+\sqrt{ }\left(1-\delta^{2}\right)}+\frac{N_{0}}{N}
$$

Proof. Let $\omega_{0}$ be the sequence having $N-N_{0}$ elements which is obtained by superposition of the $k$ almost-arithmetic progressions. Then for the discrepancy $D_{N-N_{0}}^{(0)}$ of $\omega_{0}$ we have

$$
D_{N-N_{0}}^{(0)} \leqq \sum_{i=1}^{k} \frac{N_{i}}{N-N_{0}}\left(\frac{1}{N_{i}}+\frac{\delta_{i}}{1+\sqrt{ }\left(1-\delta_{i}^{2}\right)}\right)=\frac{k}{N-N_{0}}+\sum_{=1}^{k} \frac{N_{i}}{N-N_{0}} \frac{\delta_{i}}{1+\sqrt{ }\left(1-\delta_{i}^{2}\right)}
$$


Using the same simplified denotation as in the proof of Lemma 3.1, we have, for $0<\alpha \leqq 1, A(\alpha ; N ; \omega)=A\left(\alpha ; N-N_{0} ; \omega_{0}\right)+r(\alpha)$, with $r(\alpha)$ denoting the number of the $N_{0}$ remaining elements which lie in $[0, \alpha)$. Then

$$
\begin{aligned}
\left|\frac{A(\alpha ; N ; \omega)}{N}-\alpha\right| & =\left|\frac{N-N_{0}}{N}\left(\frac{A\left(\alpha ; N-N_{0} ; \omega_{0}\right)}{N-N_{0}}-\alpha\right)+\frac{N_{0}}{N}\left(\frac{r(\alpha)}{N_{0}}-\alpha\right)\right| \\
& \leqq \frac{N-N_{0}}{N} D_{N-N_{0}}^{(0)}+\frac{N_{0}}{N} \leqq \frac{k}{N}+\sum_{i=1}^{k} \frac{N_{i}}{N} \frac{\delta_{i}}{1+\sqrt{ }\left(1-\delta_{i}^{2}\right)}+\frac{N_{0}}{N}
\end{aligned}
$$

As to the second inequality, it suffices to note that the function $f(t)=t /\left(1+\sqrt{ }\left(1-t^{2}\right)\right)$ is monotonically increasing on the interval $[0,1)$.

REMARK. By Theorem 1.1, every sufficiently long initial segment of a uniformly distributed sequence satisfies the condition of the above theorem with arbitrarily small $N_{0} / N$.

4. Application. The results of the previous section can be used to prove a quantitative form of Fejér's theorem. In addition, we get an estimate for the discrepancy of sequences which are slowly, but not too slowly, increasing. Our method can be applied to this case in a rather straightforward way, since the decomposition into almost-arithmetic progressions can be exhibited explicitly.

THEOREM 4.1. Let $f(x)$ be a monotonically increasing function defined for $x \geqq 1$ which has a continuous derivative for $x \geqq x_{0}$. Furthermore, $\lim _{x \rightarrow \infty} f(x)=\infty$, $\lim _{x \rightarrow \infty} x f^{\prime}(x)=\infty$, and $f^{\prime}(x)$ tends monotonically to zero for $x \rightarrow \infty$. Consider the sequence $\left(a_{n}\right)=(f(n)), n \geqq 1$. If $D_{N}$ denotes the discrepancy of the partial sequence of fractional parts $\left\{a_{1}\right\}, \ldots,\left\{a_{N}\right\}$, then $D_{N}=O\left(f(N) / N+1 / N f^{\prime}(N)\right)$.

Proof. Let $g(x)$ be the inverse function of $f(x)$. The following facts about $g(x)$ are pertinent: both $g(x)$ and $g^{\prime}(x)$ tend monotonically to infinity; $\lim _{x \rightarrow \infty} g^{\prime}(x) / g(x)$ $=\lim _{x \rightarrow \infty} 1 / g(x) f^{\prime}(g(x))=0$; for a fixed real number $c$, by de l'Hôpital's theorem,

$$
\begin{aligned}
\lim _{x \rightarrow \infty} \frac{g^{\prime}(x+c)}{g^{\prime}(x)} & =\lim _{x \rightarrow \infty} \frac{g(x+c)}{g(x)}=\lim _{x \rightarrow \infty} \exp (\log g(x+c)-\log g(x)) \\
& =\lim _{\xi \rightarrow \infty} \exp \left(c \frac{g^{\prime}(\xi)}{g(\xi)}\right)=1 \quad\left(\text { with } \exp (y)=e^{y}\right) .
\end{aligned}
$$

There exists a natural number $m \geqq x_{0}$ such that $f(m+1)-f(m)<1$. Furthermore, the sequence $(f(n+1)-f(n))$ is monotonically decreasing for $n \geqq m$. Put $i_{0}=[f(m)]+1$. The sequence $\left\{a_{1}\right\}, \ldots,\left\{a_{N}\right\}$ is decomposed in the following way: for fixed $i, i_{0} \leqq i \leqq[f(N)]-1$, collect all $\left\{a_{n}\right\}$ with $i \leqq a_{n}=f(n)<i+1$ into a sequence $\omega_{i}$. We shall first estimate the number $N_{i}$ of elements of $\omega_{i}$. If $f(r), f(r+1), \ldots, f(s)$ are in $[i, i+1)$, then $N_{i}=s-r+1<1 / f^{\prime}(\xi)+1<C / f^{\prime}(\xi)$ with a positive constant $C$ and $r \leqq \xi \leqq s$. Set $z=f(\xi)$, then $N_{i}<C g^{\prime}(z)<C g^{\prime}(i+1)$.

For fixed $i$ from above, let $a$ be the largest integer such that $f(a) \leqq i$, and let $b$ be the smallest integer such that $f(b) \geqq i+1$. Put $d_{1}=f(a+1)-f(a), d_{2}=f(b)-f(b-1)$. 
Then $\omega_{i}$ is an almost-arithmetic progression with parameters $\eta_{i}, \delta_{i}$ determined by $\eta_{i}+\delta_{i} \eta_{i}=d_{1}$, and $\eta_{i}-\delta_{i} \eta_{i}=d_{2}$. We have $d_{1}=1 / g^{\prime}(u)$ with $f(a)<u<f(a+1)$, and $d_{2}=1 / g^{\prime}(v)$ with $f(b-1)<v<f(b)$. Then

$$
\delta_{i}=\frac{d_{1}-d_{2}}{d_{1}+d_{2}}=\frac{g^{\prime}(v)-g^{\prime}(u)}{g^{\prime}(v)+g^{\prime}(u)}<\frac{g^{\prime}(f(b))-g^{\prime}(f(a))}{g^{\prime}(i)}
$$

since $b-a \geqq 2$, hence $v>f(b-1) \geqq f(a+1)>i$. Since $f(b)<i+2$ and $f(a)>i-1$, we get $\delta_{i}<\left(g^{\prime}(i+2)-g^{\prime}(i-1)\right) / g^{\prime}(i)$.

The elements $\left\{a_{n}\right\}$ which are not contained in a sequence $\omega_{i}$ are those for which either $a_{n}<i_{0}$ (there are $B$ of them, say) or $[f(N)] \leqq a_{n}<[f(N)]+1$ which, by the estimate for $N_{i}$, is satisfied for at most $C g^{\prime}([f(N)]+1)$ elements. By Theorem 3.1, we get

$$
\begin{aligned}
D_{N} \leqq & \frac{[f(N)]-i_{0}}{N}+\frac{1}{N} \sum_{i=i_{0}}^{[f(N)]-1} C g^{\prime}(i+1) \frac{g^{\prime}(i+2)-g^{\prime}(i-1)}{g^{\prime}(i)}+\frac{B+C g^{\prime}([f(N)]+1)}{N} \\
= & O\left(\frac{f(N)}{N}\right)+O\left(\frac{1}{N} \sum_{i=i_{0}}^{[(N)]-1}\left(g^{\prime}(i+2)-g^{\prime}(i-1)\right)\right)+O\left(\frac{g^{\prime}(f(N))}{N}\right) \\
= & O\left(\frac{f(N)}{N}\right)+O\left(\frac{1}{N} g^{\prime}([f(N)]+1)+\frac{1}{N} g^{\prime}([f(N)])+\frac{1}{N} g^{\prime}([f(N)]-1)\right) \\
& +O\left(\frac{1}{N f^{\prime}(N)}\right) \\
= & O\left(\frac{f(N)}{N}+\frac{1}{N f^{\prime}(N)}\right) .
\end{aligned}
$$

REMARK. Under the same conditions on $f(x)$, Fejér proved only the uniform distribution modulo one of the sequence $\left(a_{n}\right)$. This is, of course, contained in the above theorem because of $\lim _{x \rightarrow \infty} f(x) / x=\lim _{x \rightarrow \infty} f^{\prime}(x) / 1=0$. For a proof of Fejér's theorem, see [4, p. 237].

The classical examples of functions $f(x)$ satisfying the conditions of Theorem 4.1 are $f(x)=a x^{\sigma}, a>0,0<\sigma<1$, and $f(x)=a(\log x)^{\sigma}, a>0, \sigma>1$. For those special cases we get

COROLlaRY 1. The discrepancy $D_{N}$ of the sequence of fractional parts $\left\{a^{\sigma}\right\}$, $n=1,2, \ldots, a>0,0<\sigma<1$, satisfies $D_{N}=O\left(N^{\tau-1}\right)$ with $\tau=\max (\sigma, 1-\sigma)$.

COROLlary 2. The discrepancy $D_{N}$ of the sequence of fractional parts $\left\{a(\log n)^{\sigma}\right\}$, $n=1,2, \ldots, a>0, \sigma>1$, satisfies $D_{N}=O\left((\log N)^{1-\sigma}\right)$.

Added in proof. The first inequality in Theorem 2.1 can actually be proved by more elementary means, as will be shown in a forthcoming book by L. Kuipers and the author. The strength of the present approach lies in the fact that $\left(10^{\prime}\right)$, $\left(10^{(4)}\right),\left(11^{\prime}\right)$, and $\left(11^{(4)}\right)$, together with (4), yield an upper bound for $D_{N}$ which is attained for all values of $N$. 


\section{REFERENCES}

1. J. Cigler and G. Helmberg, Neuere Entwicklungen der Theorie der Gleichverteilung, Jber. Deutsch. Math.-Verein. 64 (1961), Abt. 1, 1-50. MR 23 \#A2409.

2. H. Niederreiter, Discrepancy and convex programming, Ann. Mat. Pura Appl. (to appear).

3. P. E. O'Neil, A new criterion for uniform distribution, Proc. Amer. Math. Soc. 24 (1970), 1-5. MR 40 \#1349.

4. G. Pólya and G. Szegö, Aufgaben und Lehrsätze aus der Analysis. Band I: Reihen, Integralrechung. Funktionentheorie, Dritte berichtigte Auflage, Die Grundlehren der math. Wissenschaften, Band 19, Springer-Verlag, Berlin and New York, 1964. MR 30 \#1219.

SOUThern Illinois University, Carbondale, Illinois 62901

Current address: Department of Mathematics, University of Illinois, Urbana, Illinois 61801 